\title{
Intravenous narcotics for premedication in outpatient anaesthesia
}

\author{
S. K. Pandit and S. P. Kothary \\ Department of Anesthesiology, University of Michigan Medical Center, Ann Arbor, Michigan, USA
}

\begin{abstract}
One hundred adult female patients scheduled for outpatient laparoscopic procedures were studied. Each patient received intravenous premedication about $30 \mathrm{~min}$ before induction of anaesthesia. The premedications were given in a double-blind random order and were either a placebo, morphine $(0.04 \mathrm{mg} / \mathrm{kg})$, meperidine $(0.35 \mathrm{mg} / \mathrm{kg})$, fertanyl $(0.75 \mu \mathrm{g} / \mathrm{kg})$ or sufentanil $(0.15 \mu \mathrm{g} / \mathrm{kg})$. All patients received a standard anaesthetic regimen. Transient light-headedness was common following narcotic injections. Overall, sufentanil was superior to the placebo and to other narcotics in its ability to reduce preoperative anxiety and to provide more satisfactory induction, maintenance and recovery from anaesthesia. The incidence of postoperative nausea, vomiting and other side effects was not higher and discharge times were not longer after sufentanil compared to the placebo group. Complete recovery as assessed by telephone interview $24-48 \mathrm{~h}$ after the operation revealed no difference between the sufentanil and the other groups. The results of this study indicate that intravenous short-acting narcotics like fentanyl or sufentanil should be considered as an alternative premedicant for anxious patients who are scheduled for outpatient surgery.
\end{abstract}

Received 9 May 1988, accepted for publication 6 January 1989

Key words: Anesthesia: outpatient; premedication: intravenous, narcotics - fentanyl, merperidine, morphine, sufentanil.

The use of preanaesthetic medication is still a matter of much controversy in outpatient anaesthesia practice. Ambulatory surgical patients have little prior contact with their anaesthesiologist, have little time to get adjusted to the hospital environment and are thus often very anxious when they arrive in the preoperative room. Some of these patients might benefit from being given a pharmacological premedication in addition to psychological support.

It is not uncommon for anaesthesiologists to administer a small dose of short-acting narcotic during induction of anaesthesia as a part of the overall anaesthesia care plan. The narcotic is expected to reduce the minimum alveolar concentration (MAC) and thus reduce the need for inhalation anaesthetic agents. In addition, the analgesic effect of the intraoperative narcotic may extend to the immediate postoperative period to provide smoother recovery. We wondered if it would be more beneficial for the patient if we administered the same small dose of a short-acting narcotic as an intravenous premedication instead of using it during the induction of anaesthesia. Because of the reported "euphoric" effect of some of the narcotics, these premedicants might reduce patients' preoperative anxiety, in addition to providing smoother induction, maintenance and recovery from anaesthesia.

The aim of the study was to compare comparable doses of morphine, meperidine, fentanyl and sufentanil with a placebo (normal saline) given intravenously as preanaesthetic medication for ambulatory surgical patients. We assessed the effects of the premedicants on anxiety, sedation, ease of anaesthetic induction, maintenance, requirement for postoperative analgesics, recovery time and frequency of side effects.

\section{PATIENTS AND METHODS}

Prior approval from the Institutional Ethical Committee concerning use of human subjects for research was obtained. Each patient gave written informed consent before participating in the study.

The study included 100 adult female patients (ASA I or II) between the ages of 18 and 45 years who were scheduled for an outpatient laparoscopic procedure (diagnostic or for tubal ligation) under general anaesthesia. Drug-dependent individuals, patients who had received drugs that affect the central nervous system within the last 7 days and patients with significant involvement of cardiac, respiratory, metabolic or central nervous systems were excluded. The assigned anaesthesiologist performed the routine preanaesthetic evaluation in the preoperative waiting room. One of the investigators then approached the patient and obtained informed consent for the study.

After the baseline assessment (see below), approximately $30 \mathrm{~min}$ prior to the induction of anaesthesia, one of the four active medications or a placebo was injected through a previously secured intravenous route. The doses of premedicants used were morphine, 0.04 $\mathrm{mg} \cdot \mathrm{kg}^{-1}$, meperidine $0.35 \mathrm{mg} \cdot \mathrm{kg}^{-1}$, fentanyl $0.75 \mu \mathrm{g} \cdot \mathrm{kg}^{-1}$, sufentanil $0.15 \mu \mathrm{g} \cdot \mathrm{kg}^{-1}$ and placebo (normal saline) $2 \mathrm{ml}$. There were 20 
cases in each group. All medications were calculated and dispensed in equal volume $(2 \mathrm{ml})$ by the same nurse throughout the study. This nurse was not involved in any of the subsequent assessments.

Anxiety was assessed subjectively, utilizing a visual analogue scale, which consisted of a $100-\mathrm{mm}$ vertical line, where ' 0 ' was marked as "no anxiety at all" and 100 was marked as "extreme anxiety". After receiving a full explanation of the scale before the premedication, patients were asked to place a mark on the scale to denote the level of current anxiety. Each patient assessed her own anxiety before premedication, $15 \mathrm{~min}$ after premedication, and again just before induction of anaesthesia in the operating room.

Arterial pressure (systolic, diastolic and mean) and pulse rates were recorded before premedication and at 15 -min intervals thereafter until the time of induction by means of an automatic noninvasive device with a printer. Respiratory rates were also recorded. Vital signs continued to be recorded by the automatic device during induction of anaesthesia (every minute), maintenance period (every $3-5 \mathrm{~min}$ ) and in the recovery room (every $15 \mathrm{~min}$ ). Each patient was closely observed following the premedication, and the frequency of side affects such as pain on injection, dizziness, nausea and slecpiness immediately after premedication were noted following direct questioning. Grades of sedation were noted before premedication, 15 min later and just before induction of anaesthesia utilizing a scoring system ( 1 ) in which $1=$ no sedation, $2 \simeq$ relaxed but not sleepy, $3=$ sleepy and easily arousable by a light touch or gentle command, $4=$ asleep and arousable by a strong stimulus, and $5=$ patient unable to communicate.

The assigned anaesthesiologist (not part of the study and blind to the premedication given) gave overall evaluation of the effectiveness of the premedicant as excellent, good, fair, or poor just prior to the induction of anaesthesia using sleepiness, anxiety and overall calmness as the criteria. Anaesthetic technique was standardized in all cases. After preoxygenation, induction of anaesthesia was achieved with thiamylal, $4 \mathrm{mg} \cdot \mathrm{kg}^{-1}$ and vecuronium, $0.1 \mathrm{mg} \cdot \mathrm{kg}^{-1}$. Following positive pressure ventilation via a face mask with oxygen and isoflurane $(2 \%)$ for $4 \mathrm{~min}$ (or until full paralysis was demonstrated by a peripheral nerve stimulator), the trachea was intubated. Anaesthesia

\section{Grades of Sedation}

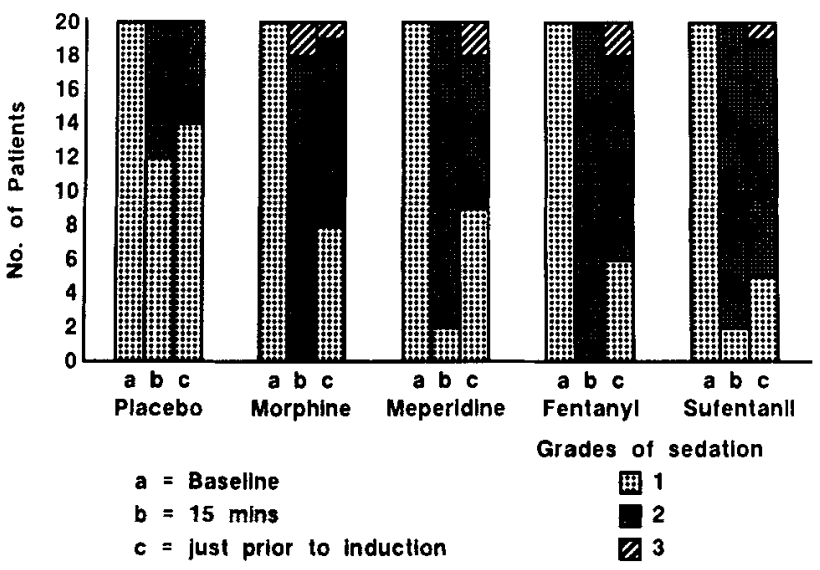

Fig. 1. Showing distribution of cases with various grades of sedation from the five groups. Grade $1=$ no sedation, Grade $2=$ relaxed but not asleep, Grade $3=$ sleepy and easily arousable. (There were no cases in Grade 4 or 5). ANOVA and multiple comparison with Scheffe allowances showed, at $15 \mathrm{~min}$ placebo $P<0.001$ against all other groups, but just prior to induction placebo $P<0.01$ only against fentanyl and sufentanil.
Table I

Demography and duration of anesthesia (mean \pm s.d.).

\begin{tabular}{lccccc}
\hline Premedicant & $\mathbf{n}$ & $\begin{array}{c}\text { Age } \\
\text { (years) }\end{array}$ & $\begin{array}{c}\text { Weight } \\
(\mathrm{kg})\end{array}$ & $\begin{array}{c}\text { Height } \\
(\mathrm{cm})\end{array}$ & $\begin{array}{c}\text { Duration } \\
(\mathrm{min})\end{array}$ \\
\hline Morphine & 20 & $30.1 \pm 6.2$ & $61.2 \pm 8.3$ & $64.1 \pm 2.3$ & $53.7 \pm 18.7$ \\
Meperidine & 20 & $30.9 \pm 4.5$ & $65.3 \pm 16.5$ & $64.9 \pm 2.7$ & $51.5 \pm 16.3$ \\
Fentanyl & 20 & $29.9 \pm 6.6$ & $63.7 \pm 10.6$ & $64.9 \pm 3.2$ & $54.0 \pm 19.7$ \\
Sufentanil & 20 & $29.0 \pm 3.9$ & $63.4 \pm 9.3$ & $64.7 \pm 2.1$ & $52.0 \pm 8.3$ \\
Placebo & 20 & $30.0 \pm 4.9$ & $61.1 \pm 10.2$ & $64.2 \pm 2.2$ & $48.8 \pm 11.9$
\end{tabular}

No significant differences among the groups (ANOVA).

was maintained with nitrous oxide and oxygen $(4: 2 \mathrm{l} / \mathrm{min})$, and isoflurane (usually $1 \%$ ). Ventilation was controlled mechanically and additional vecuronium ( $2 \mathrm{mg}$ increments) was administered if needed. No additional narcotic was administered unless excessive tachycardia (heart beat $100 \mathrm{bpm}$ for $5 \mathrm{~min}$ ) and/or hypertension (systolic pressure more than $150 \mathrm{mmHg}(20.0 \mathrm{kPa})$ for $5 \mathrm{~min}$ ) were present. In that case, fentanyl in $25 \mu \mathrm{g}$ increments was injected. A standard dose of droperidol $(0.625 \mathrm{mg}$ ) was administered intravenously in all cases just after tracheal intubation as a prophylactic antiemetic agent. An orogastric tube (Salem sump) was placed in each patient soon after tracheal intubation to decompress the stomach. The stomach tube was left in place until just before tracheal extubation. At the end of the procedure, the effect of vecuronium was reversed with appropriate doses of neostigmine and glycopyrrolate.

In the recovery room, we rated the recovery score (Aldrete Score, $0-10)$ on arrival, arterial pressure, heart rate, and respiratory rate every $15 \mathrm{~min}$, the time to complete orientation (day, date and date of birth), the time to ambulation and time to discharge. Patients were discharged from the recovery room following strict clinical criteria (Wetchler criteria) (2). These criteria consisted of stable vital signs, absence of respiratory distress, presence of gag and cough reflex, ability to walk to the bathroom, ability to void and tolerance to oral fluid intake. The frequency of side effects during recovery was recorded every $15 \mathrm{~min}$. Any incidence of nausea and vomiting, excessive pain (moderate or severe, defined as requiring intravenous pain medication), dizziness, headache or other symptoms were recorded after direct questioning. In cases of excessive nausea or vomiting (on nurse's judgment) perchlorperazine $5 \mathrm{mg}$ i.v. was given; in case of moderate to severe pain, fentanyl (25 $\mu \mathrm{g}$ i.v.) was administered. The dose was repeated once if necessary. Oral pain medication (acetaminophen or ibuprofen) was given when the patients with pain could tolerate oral intake. Grades of sedation were also noted every $15 \mathrm{~min}$, following the same scale used in the preoperative period. Grades 4 and 5 were considered excessive drowsiness.

Table 2

Side effects following i.v. premedication (\%).

\begin{tabular}{lcccc}
\hline Premedicant & $\mathrm{n}$ & $\begin{array}{c}\text { Discomfort } \\
\text { at i.v. site }\end{array}$ & Drowsy & Lightheaded \\
\hline Morphine & 20 & $60 \dagger$ & 20 & $50^{*}$ \\
Meperidine & 20 & 32 & 21 & $53^{*}$ \\
Fentanyl & 20 & 20 & 25 & $60^{*}$ \\
Sufentanil & 20 & 10 & 30 & $50^{*}$ \\
Placebo & 20 & 15 & 0 & 5 \\
\hline
\end{tabular}

$\dagger$ Morphine significantly different from fentanyl, sufentanil and placebo, but not from meperidine (chi-square test).

* Placebo significantly different from all other groups (chi-square test). 


\section{Statistical analysis}

We used a two-factor ANOVA (profile analysis) for repeated measures to compare means within and between groups. Where we found interaction in time and group effects, we also used pair-wise $t$-tests, with Bonferroni correction, to test for significance within the groups and one-factor ANOVA to compare means between groups at given times. This allowed us to test for interactions between time and group effects as well as testing for each effect individually. Results were considered significant if the $P$-value was 0.05 or less.

\section{RESULTS}

Demographic parameters of age, weight, height, and duration of anaesthesia (approximately $60 \mathrm{~min}$ ) were comparable in the five groups, and there were no significant statistical differences (ANOVA) (Table 1). Approximately $50 \%$ of the patients complained of transient light-headedness after narcotic premedication and 10 to $30 \%$ felt drowsy, but these symptoms did not last long and both symptoms were clinically unimportant. However, in statistical term, the placebo group had significantly less light-headedness than all other groups. Both morphine and meperidine caused a higher incidence of discomfort (usually mild itchiness and burning) at the injection site $(60 \%$ and $32 \%$, respectively) compared to the placebo (15\%), fentanyl $(20 \%)$ and sufentanil $(10 \%)$ (Table 2). Regarding local discomfort, the morphine group was statistically different from the fentanyl, sufentanil and placebo groups but not from the meperidine group. No clinically significant respiratory depression, hypotension, tachycardia, bradycardia or chest rigidity were noted following any premedication.

Analyzing the anxiety measure with the two-factor ANOVA, we found there to be a significant interaction between time and group effects. Therefore we proceeded to assess the effects individually using a pairwise $t$ test with the Bonferroni correction to compare the mean anxiety levels within each drug group between the three time points, and then using a one-factor ANOVA we compared the mean anxiety levels at each time point between drug groups. Our findings are displayed in Table 3.

Within all groups, except placebo, there was a significant difference between anxiety levels at baseline and at $15 \mathrm{~min}$. Comparing baseline to anxiety levels prior to induction, there was a significant difference in the fentanyl and sufentanil groups using the Bonferroni correction.

Between groups at baseline there was a significant difference between mean anxiety level of the meperidine and fentanyl groups. At the 15-min measurement the morphine, meperidine, fentanyl and sufentanil groups had significantly lower mean anxiety levels than the placebo group. Just prior to induction only the sufentanil group had a mean anxiety level significantly lower than placebo.

Similarly, $15 \mathrm{~min}$ after premedication, grades of sedation were significantly higher (indicating more sedation) in all active medication groups compared to the placebo group. However, just before induction of anaesthesia, only the fentanyl and sufentanil groups were significantly different from placebo in this respect (Fig. 1). The anesthesiologists' overall evaluation of premedication favoured sufentanil $(70 \%$ good to excellent) compared to morphine $(45 \%)$, meperidine $(45 \%)$, fentanyl $(40 \%)$ and the placebo $(20 \%)$ (Table 4). In this respect, only sufentanil was significantly different (statistically) to placebo.

Satisfactory induction of anaesthesia was defined as a case that required no additional thiamylal. Satisfactory maintenance was a case that did not require any additional narcotic (fentanyl) to maintain anaesthesia. Patients receiving sufentanil premedication had satisfactory induction in $100 \%$ of the cases and satisfactory maintenance of anaesthesia in $95 \%$ of the cases (Table 5). This table thus in effect shows the number of patients who required no additional thiamylal during induction and no additional fentanyl during maintenance. Only the data on sufentanil were significantly different from the placebo results both during induction and maintenance.

Table 3

Anxiety scores and premedication (mean \pm s.d.) (percent reduction from baseline in parenthesis).

\begin{tabular}{lcccc}
\hline Premedicant & $\mathrm{n}$ & Baseline & $15 \mathrm{~min}$ & Prior to induction \\
\hline Morphine & 20 & $50.7 \pm 26.91$ & $*+28.3 \pm 24.62(-45 \%)$ & $39.6 \pm 29.51(-22 \%)$ \\
Meperidine & 20 & $\dagger 44.7 \pm 24.57$ & $*+21.3 \pm 19.46(-52 \%)$ & $30.9 \pm 29.29(-30 \%)$ \\
Fentanyl & 20 & $+65.1 \pm 26.32$ & $*+32.7 \pm 19.23(-50 \%)$ & $45.5 \pm 27.88^{*}(-30 \%)$ \\
Sufentanil & 20 & $52.0 \pm 26.3$ & $*+27.1 \pm 23.91(-48 \%)$ & $* * 29.6 \pm 28.68^{*}(-43 \%)$ \\
Placebo & 20 & $51.9 \pm 27.02$ & $43.5 \pm 29.20(-16 \%)$ & $48.7 \pm 26.89(-6 \%)$ \\
\hline
\end{tabular}

$\dagger$ ANOVA $P<0.05$ between meperidine and fentanyl.

+ ANOVA $P<0.05$ when compared to placebo.

** ANOVA $P<0.05$ when compared to placebo.

* $P \leq 0.05$ Pairwise t-test with Bonferroni correction when compared to baseline. 
Table 4

Anesthesiologists' overall evaluation of premedication (\%).

\begin{tabular}{lcc}
\hline Premedicant & Excellent/good & Fair/poor \\
\hline Morphine & 45 & 55 \\
Meperidine & 45 & 55 \\
Fentanyl & 40 & 60 \\
Sufentanil & $* 70$ & $* 30$ \\
Placebo & 20 & 80 \\
\hline
\end{tabular}

* Sufentanil significantly different from placebo (chi-square test).

The Aldrete recovery room scores on arrival in the recovery room were similar in all groups (range $8.5 \pm$ 0.8 to $8.7 \pm 1.3$ ) and were statistically insignificant. The recovery times (time to complete orientation, time to ambulation and time to discharge) are shown in Table 6 . These times were not significantly different among the various groups (ANOVA).

The frequency of side effects is shown in Table 7. There was a trend to greater nausea and vomiting in the morphine and meperidine groups (25 and 20\%, respectively), but the incidence in the sufentanil group was the same as in the placebo group $(10 \%)$. The fentanyl group had a $15 \%$ incidence of nausea and vomiting. Differences between the groups regarding the incidence of nausea and vomiting, however, did not reach statistical significance. The frequency of excessive drowsiness in the recovery room was similar among the groups and was not statistically different. The need for intravenous and oral analgesics in the recovery room was also not different among the groups.

Patients were telephoned at home 24 and $48 \mathrm{~h}$ after the operation and were asked, "When did you feel normal?" Between 80 and $95 \%$ of patients in various groups said they felt normal by the second postoperative day (the day after the operation). There was no significant difference among the groups regarding recovery time, and results are not shown. None of the patients suffered from other significant side effects, such as protracted nausea/vomiting, dizziness, drowsiness and muscle pain.

Table 5

Quality of anesthesia (\%) after premedications.

\begin{tabular}{lcc}
\hline Premedicant & Satisfactory induction & Satisfactory maintenance \\
\hline Morphine & $95^{*}$ & 80 \\
Meperidine & 85 & 75 \\
Fentanyl & $95^{*}$ & 85 \\
Sufentanil & $100^{*}$ & $95 \dagger$ \\
Placebo & 65 & 65
\end{tabular}

* Placebo was significantly different from fentanyl, sufentanil and morphine (chi-square test).

$\uparrow$ Placebo significantly different than sufentanil (chi-square test).
Table 6

Recovery time (in min) for premedication patient groups (mean \pm s.d.).

\begin{tabular}{lccc}
\hline Premedicant & Orientation & Ambulation & Discharge \\
\hline Morphine & $21.4 \pm 6.63$ & $148.4 \pm 49.33$ & $210.3 \pm 65.84$ \\
Meperidine & $19.2 \pm 6.54$ & $135.2 \pm 30.02$ & $187.0 \pm 50.87$ \\
Fentanyl & $19.9 \pm 7.41$ & $145.8 \pm 51.73$ & $187.9 \pm 67.73$ \\
Sufentanyl & $19.7 \pm 8.19$ & $134.2 \pm 44.05$ & $181.5 \pm 62.01$ \\
Placebo & $20.2 \pm 7.69$ & $156.0 \pm 54.04$ & $200.0 \pm 69.12$ \\
\hline
\end{tabular}

No significant difference among groups (ANOVA).

Arterial pressures, heart rates and respiratory rates following premedication, during induction and maintenance of anaesthesia and also during the recovery room period were remarkably stable in all groups, and are not shown. Arterial pressure and heart rate increased transiently and significantly in all groups following endotracheal intubation.

\section{DISCUSSION}

There are a number of papers in the literature which suggest that judicious use of premedicants for outpatient surgical patients does not delay recovery time $(3,4)$. However, the common benzodiazepine, diazepam, is usually avoided for outpatients because of its long elimination half-life and its potential for pain on injection, tissue damage and thrombophlebitis. Lorazepam is too long acting an agent to be useful in outpatient anaesthesia. The new water-soluble, shortacting midazolam is a good anxiolytic and not painful on injection, and is thus suitable for outpatient use (5). Of the narcotics we compared with a placebo as intravenous premedicants, the two short-acting agents, fentanyl and sufentanil, especially sufentanil, stood out as the most promising for the purpose. Sufentanil not only reduced preoperative patient anxiety but also provided smoother induction and maintenance, without increasing postoperative side effects such as nausea, and without delaying discharge time.

The equianalgesic doses of narcotics, especially fentanyl versus sufentanil, have been a matter on which there are conflicting views $(6,7)$. Sufentanil has been

Table 7

Side effects in the recovery room $(\%)$.

\begin{tabular}{lccc}
\hline Premedicant & Nausea/vomiting & Drowsiness & Excessive pain \\
\hline Morphine & 25 & 15 & 45 \\
Meperidine & 20 & 20 & 45 \\
Fentanyl & 15 & 15 & 45 \\
Sufentanil & 10 & 15 & 40 \\
Placebo & 10 & 15 & 45 \\
\hline
\end{tabular}

No significant difference between groups (chi-square tests). 
claimed to be five times as potent as fentanyl, although recent studies have suggested even higher potency. On the other hand, equipotency of the narcotics as antianxiety agents is unknown. Regarding equipotent doses of morphine, meperidine and fentanyl, Marshall \& Wollman (8) stated in a highly respected pharmacology textbook that "intravenous doses of 1 to 2 $\mathrm{mg}$ of morphine, 10 to $25 \mathrm{mg}$ of meperidine and 0.05 to $0.1 \mathrm{mg}$ of fentanyl are approximately equipotent". The doses we used were within these recommendations.

Many anaesthesiologists believe that morphine and meperidine premedication often cause euphoria, which helps in decreasing patient anxiety. We did not see any sign of euphoria with any of these agents in our study. Not only were these two long-acting agents less effective in reducing preoperative anxiety, but their contribution to the improved quality of induction and maintenance was also less clearcut. Furthermore, there was a trend towards a higher incidence of nausea and vomiting in patients who received morphine and meperidine, compared to those who received the placebo, although with the number of cases in our study this difference did not reach the level of statistical significance. Conner et al. (9) compared morphine and meperidine (in much higher doses than ours) as intravenous premedicants, and reported findings very similar to ours. They found neither meperidine nor morphine to be particularly good surgical premedicants. Neither caused significant euphoria or relief from anxiety, and the incidence of nausea and vomiting after premedication was high.

The incidence of postoperative nausea and vomiting in all groups was low in our study. Previous studies in the same patient population (young females undergoing a laparoscopic procedure), with a very similar anaesthetic technique, showed a very high incidence $(50-60 \%)$ of postoperative nausea and vomiting $(10)$. In the present study we used droperidol $0.625 \mathrm{mg}$ i.v. as a prophylactic antiemetic agent at the time of induction of anaesthesia. We believe that pretreatment with droperidol (11), as well as routine decompression of the stomach during operation, were the reasons for the lower incidence and lower severity of nausea and vomiting.

About $50 \%$ of the patients receiving intravenous narcotic premedication, including sufentanil, complained of transient light-headedness immediately after the injection. Although this side effect lasted only 1 or $2 \mathrm{~min}$, it might be considered a drawback of intravenous premedication. We did not notice any obvious rigidity, excessive tachycardia or hypotension after the premedicants. The high incidence of discomfort during i.v. injection of morphine and meperi- dine was surprising, but these discomforts were minor in nature and were obtained only on direct questioning immediately after injection.

Dispensing the small dose of sufentanil 10-20 $\mu \mathrm{g}$ in the commercially available concentration would appear cumbersome and even dangerous (because of the possibility of a mistake in delivering small doses); to avoid these problems we recommend routine dilution of sufentanil at 5-10 $\mu \mathrm{g} \cdot \mathrm{ml}^{-1}$ to be used only in the preoperative waiting room (for outpatient or inpatients) and not on the inpatient floor.

With the introduction of the water-soluble, shortacting benzodiazepine, midazolam (Roche), the need for an intravenous narcotic premedication might appear to have receded. However, midazolam does not possess any analgesic property; thus, in cases where a potent analgesic is a necessity during the operation, sufentanil premedication might still have an important place in outpatient anesthesia. Besides, the profound anterograde amnesic effects of midazolam (5) may be considered as a disadvantage in some outpatient surgical patients.

We conclude from our study that if an outpatient needs a pharmacological premedication, an intravenous, short-acting narcotic, should be considered as an alternative. Sufentanil $0.15 \mu \mathrm{g} \cdot \mathrm{kg}^{-1}$ given intravenously is superior to a placebo and to other common narcotics as a premedicant.

\section{ACKNOWLEDGEMENTS}

The assistance of Ms Marilyn Fulton, LPN, the waiting room nurse, who dispensed the premedicants from a random list, is gratefully acknowledged. We are grateful to Dale B. Tuttle of the Department of Anesthesiology Research and Prof. M. Anthony Schork, Ph.D., Department of Biostatistics for their help with the statistical analysis.

Presented at the annual meetings 1986 of the American Society of Anesthesiologists, Las Vegas and the Society for Ambulatory Anesthesia, Williamsbury, USA.

\section{REFERENCES}

1. Pandit S K, Heisterkamp D V, Cohen P J. Further studies of the antirecall effect of lorazepam: a dose-time-effect relationship. Anesthesiology 1976: 45: 495-500.

2. Wetchler B V. Problem solving in the postanaesthesia care unit. In: Wetchler B V, ed. Anesthesia for ambulatory surgery. Philadelphia: J. B. Lippincott Company, 1985: 275-320.

3. Clark A J, Hurtig J B. Premedication with meperidine and atropine does not prolong recovery to street fitness after outpatient surgery. Can Anaesth Soc $\mathcal{J}$ 1981: 28: 390-393.

4. Jakobsen H, Hertz J B, Johansen J R, Hansen A, Kolliker K. Premedication before day surgery: a double-blind comparison of diazepam and placebo. Br 7 Anaesth 1985: 57: 300-305.

5. Khanderia U, Pandit S K. Use of midazalam hydrochloride in anesthesia. Clin Pharm 1987: 6: 533-547.

6. Bovill J G, Sebel P S, Stanley T H. Opioid analgesics in anes- 
thesia with special reference to their use in cardiovascular anesthesia. Anesthesiology 1984: 61: 731-755.

7. Flacke J W, Bloor B C, Kripke B J et al. Comparison of morphine, meperidine, fentanyl and sufentanil in balanced anesthesia: a double-blind study. Anesth Analg 1985: 64: 897-910.

8. Marshall B E, Wollman H. General anesthetics. In: Gillman A G, Goodman L S, Rall T W, Murad F, eds. The pharmacological basis of therapeutics, 7 th edn. New York: MacMillan Publishing Co., 1985: 276-30I.

9. Conner J T, Weldon B J, Katz R L. Meperidine and morphine as intravenous surgical premedicants. Can Anaesth Soc $\mathcal{J}$ 1977: 24: $559-564$

10. Pandit S K, Kothary S P, Pandit U A, Mathai M K. Comparison of fentanyl and butorphanol for outpatient anaesthesia. Can Anaesth Soc 7 1987: 34: 130-134.
11. Pandit S K, Kothary S P, Pandit U A. Antiemetic efficacy of oral metoclopramide versus intravenous droperidol for outpatient laparoscopic procedures. Anesthesiology 1987: 67: A425.

Address:

Sujüt K. Pandit, M.D.

Department of Anesthesiology

University of Michigan Medical Center

1 G323 University Hospital

East Medical Center Drive

Ann Arbor, Michigan 48109-0048

U.S.A. 